\title{
Enumerating Graph Embeddings and Partial-Duals by Genus and Euler Genus
}

\author{
Jonathan L. Gross ${ }^{\dagger}$ and Thomas W. Tucker ${ }^{\ddagger}$ \\ ${ }^{\dagger}$ Department of Computer Science, Columbia University, New York, NY 10027 USA \\ Email: gross@cs.columbia.edu \\ ${ }^{\ddagger}$ Department of Mathematics, Colgate University, Hamilton, NY 13346, USA \\ Email: ttucker@colgate.edu
}

Received: September 2, 2020, Accepted: November 16, 2020, Published: November 20, 2020

The authors: Released under the CC BY-ND license (International 4.0)

ABSTRACT: We present an overview of an enumerative approach to topological graph theory, involving the derivation of generating functions for a set of graph embeddings, according to the topological types of their respective surfaces. We are mainly concerned with methods for calculating two kinds of polynomials:

1. the genus polynomial $\Gamma_{G}(z)$ for a given graph $G$, which is taken over all orientable embeddings of $G$, and the Euler-genus polynomial $\mathcal{E}_{G}(z)$, which is taken over all embeddings;

2. the partial-duality polynomials ${ }^{\partial \mathcal{E}_{G}^{*}}(z),{ }^{2} \mathcal{E}_{G}^{\times}(z)$, and ${ }^{\partial \mathcal{E}_{G}^{* \times *}}(z)$, which are taken over the partial-duals for all subsets of edges, for Poincaré duality $(*)$, Petrie duality $(\times)$, and Wilson duality $(* \times *)$, respectively.

We describe the methods used for the computation of recursions and closed formulas for genus polynomials and partial-dual polynomials. We also describe methods used to examine the polynomials pertaining to some special families of graphs or graph embeddings, for the possible properties of interpolation and log-concavity.

Mathematics Subject Classifications: 05A05; 05A15; 05C10; 05C30; 05C31

Key words and phrases: Genus polynomial; Graph embedding; Log-concavity; Partial duality; Production matrix; Ribbon graph; Monodromy

\section{Introduction}

We are concerned with two classes of enumerative invariants regarding the embeddings of graphs in surfaces of arbitrarily high genus or crosscap number. One class is the embedding polynomials of a graph, as initiated by [24]. The other is the partial-duality polynomials of a graph embedding, which are recently introduced invariants [30-32], in which the authors have taken a special interest.

In this overview, we encounter three different paradigms for specifying a graph embedding.

1. Rotation systems - the dominant paradigm for combinatorial specification of embeddings; introduced by Heffter [41] and Edmonds [17].

2. Ribbon graphs - used by Chmutov [16] to give a geometric definition of partial Poincaré duals.

3. Monodromy - the dominant paradigm in algebraic map theory; introduced by Jones and Singerman [43]

In general, we allow a graph to have loops and multi-edges. Each edge is construed to have two half-edges (that meet midway along the length of the edge). We label the edges of a graph by the integers $1,2, \ldots, n$, and we denote the half-edges of edge $j$ by $j^{+}$and $j^{-}$. A direction of traversal of an edge may be given by specifying the half-edge that is traversed first. A graph is taken to be connected, unless it is evident from context that we mean otherwise. The genus of an orientable surface is denoted by $\gamma(S)$. The orientable surface of genus $j$

\footnotetext{
† Jonathan Gross is supported by Simons Foundation Grant No. 315001

$\ddagger$ Thomas Tucker is supported by Simons Foundation Grant No. 317689
} 
is denoted $S_{j}$ and the non-orientable surface of crosscap-number $k$ is denoted $N_{k}$. Some prior familiarity with topological graph theory beyond planarity is assumed. Some recommended sources are [37] or [64].

Section 2 describes the paradigm of rotation systems. Section 3 presents methods used in their derivations for the genus polynomials of what are called linear sequences and ring-like sequences of graphs. Section 4 describes methods for other kinds of graphs, including some important non-linear families for which family-wide genus polynomial formulas are known. Section 5 discusses some possible structural attributes of these embeddingrelated polynomials, especially log-concavity. Section 6 describes the paradigms of monodromy and ribbon graphs. Section 7 discusses all three kinds of duals of graph embeddings and their corresponding partial duals. Section 8 presents formulas for the partial-dual polynomials of several sequences of ribbon graphs. Section 9 suggests a number of problems for investigation.

We have italics on various technical terms to indicate that their definitions appear later, usually along with some developmental detail.

\section{Rotation systems}

A rotation at a vertex $v$ of an oriented embedding of a graph $G$ is a cyclic list of the half-edges incident at $v$, in the order consistent with the orientation of the surface. The collection of rotations, one at every vertex, is called a rotation system for $G$, commonly denoted by $\rho$. It has long been known that the oriented embeddings of a graph $G$ are in bijective correspondence with the set of all possible rotation systems for $G$. This has the following immediate implication:

Proposition 2.1. The total number of oriented embeddings of a graph $G$ equals

$$
\prod_{v \in V(G)}(\delta(v)-1) !
$$

where $V(G)$ is the vertex set of $G$ and $\delta(v)$ is its valence (or degree).

When we compose a rotation system $\rho$ for an embedded graph with the full involution (commonly denoted by $\lambda$ ) that reverses the directions of the edges, the result is a permutation whose cycles correspond to the boundary walks for the faces of the embedding. Thus, the embedding could be reconstructed by fitting a polygon to each of those cycles and pasting the polygons together.

Proposition 2.2. The face-boundary walks of an embedding with rotation system $\rho$ are given by the composition $\rho \lambda$. (The composition $\rho \lambda$ means $\lambda$ is applied first.)

Given an embedding (called the primal embedding) of a graph in a surface, we form a Poincaré dual of $G$ and its embedding as follows:

1. place a dual vertex in the interior of each region of the primal embedding;

2. through each primal edge $e$ (i.e., of the graph $G$ ), draw a dual edge $e^{*}$ that joins the dual vertex on one side of $e$ to the dual vertex on its other side. If a single face of the primal embedding is incident on both sides of edge $e$, then the dual edge $e^{*}$ is a loop.

According to [1], knowledge of the duality between the cube and the octahedron, and between the dodecahedron and the icosahedron goes back to the "fifteenth book of Euclid", which [1] estimates as having originated in the sixth century C.E. Poincaré developed algebraic consequences of a generalization of this topological form of duality to higher dimensions.

Corollary 2.1. The rotation system for the Poincaré dual of an embedding with rotation system $\rho$ is given by the composition $\rho \lambda$.

The minimum genus of a graph $G$, denoted $\gamma_{\min }(G)$ is the smallest genus of a surface in which $G$ can be embedded. The maximum genus, denoted $\gamma_{\max }(G)$ is the largest genus of a surface in which $G$ can be cellularly embedded, which means embedded so that the interior of every region is homeomorphic to an open disk. The genus polynomial of the graph $G$, as defined by [24], is the formula

$$
\Gamma_{G}(z)=\sum_{j=\gamma_{\min }(G)}^{\gamma_{\max }(G)} g_{j}(G) z^{j},
$$

where $g_{j}(G)$ is the number of embeddings of $G$ in the orientable surface $S_{j}$. Two analogous polynomials have been defined subsequently $[7]$ :

1. the crosscap-number polynomial $\mathcal{X}_{G}(z)$, which enumerates embeddings of $G$ in non-orientable surfaces $N_{j}$, according to crosscap number $j$; and

2. the Euler-genus polynomial $\mathcal{E}_{G}(z)$, which enumerates embeddings of $G$ in all surfaces, according to Euler genus. 


\section{Genus polynomial formulas for linear graph families}

This section begins with a description of methods that have been used for derivation of formulas for the genus of graphs that are grouped into a linear sequence. We follow this with discussion of ring-like sequences, in which each graph is obtained by some kind of self-amalgamation of a graph in a corresponding linear sequence.

Two embeddings of a graph $G$ are topologically equivalent if one can be obtained from the other by ambient isotopy. When a graph is embedded in a surface, the induced rotation at a vertex means the cyclic ordering of half-edges that are encountered as we traverse a small circle surrounding the vertex. If the surface is oriented, then we traverse each such circle in the direction consistent with the orientation. Then the induced rotation system is the set of such vertex rotations. It has long been known that two embeddings of a graph are topologically equivalent if and only if their induced rotation systems are the same.

If the embedding surface is non-orientable, then we choose a spanning tree $T$, and then choose the vertex rotations so that any two vertex rotations are consistent whenever the two vertices are endpoints of the same tree edge. A co-tree edge in either embedding of $G$ is said to be twisted whenever the rotations at its endpoints are inconsistent. Then two embeddings are topologically equivalent if and only if they have not only the same rotation systems but also the same sets of twisted edges, relative to a spanning tree T. See [37] for further details.

\subsection{Linear sequences of graphs}

A linear sequence of graphs is a sequence constructed recursively, as now introduced via an example. By a ladder graph (or simply ladder), we mean the cartesian product graph of a path graph with the complete graph $K_{2}$. The genus polynomials of ladder graphs were derived by [19]. Figure 1 illustrates the ladder sequence and the ingredients of their recursive construction.

(a)

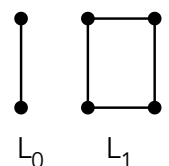

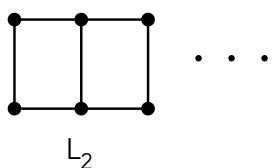

(b)

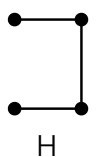

Figure 1: (a) Sequence of ladder graphs. (b) Subgraph for the recursion.

The base graph for a recursive construction is the ladder $L_{0}$, a vertical copy of $K_{2}$. We construct the ladder $L_{n}$ by amalgamating the two vertices at the left end of the subgraph $H$ to the two endpoints of the rightmost rung of the ladder $L_{n-1}$.

According to Formula (1), the ladder $L_{n}$ has $2^{n-2}$ embeddings. In order to construct their genus polynomials, the key step is partitioning the embeddings into two types. An embedding of type $d$ has two different faceboundary walks incident on its rightmost edge. An embedding of type $s$ has the same face-boundary walk twice incident on that edge. We define the numbers $d_{j}\left(L_{n}\right)$ and $s_{j}\left(L_{n}\right)$ to be the numbers of type- $d$ and type- $s$ embeddings of $L_{n}$, of genus $j$, respectively, which leads to the type- $d$ and type-s partial genus polynomials

$$
\begin{aligned}
& D_{L_{n}}(z)=\sum_{j=0}^{n} d_{j}\left(L_{n}\right) z^{j} \\
& S_{L_{n}}(z)=\sum_{j=0}^{n} s_{j}\left(L_{n}\right) z^{j} .
\end{aligned}
$$

Figure 2 illustrates the four ways that subgraph $H$ can be added to a ladder of type- $d$ or of type- $s$. We seek to convert such a figure into a simultaneous recursion.

(d)
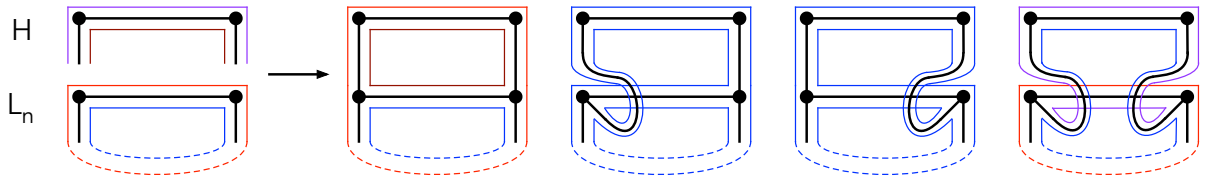

(s)
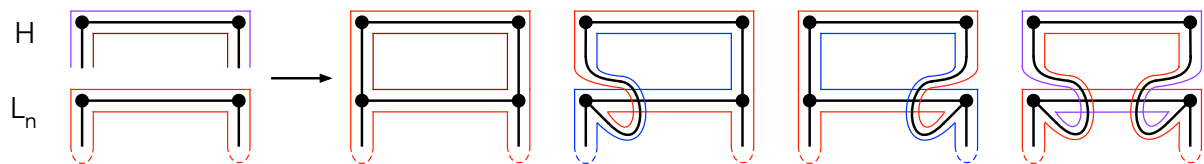

Figure 2: (d) Adding $H$ to a type- $d$ ladder. (s) Adding $H$ to a type- $s$ ladder. 
In row (d), we see that the first and fourth ways of adding subgraph $H$ to ladder $L_{n}$ add two vertices, three edges, and one face, for no change to the Euler characteristic or genus. We see also that two different face-boundary walks are incident on the new rung, indicating a type- $d$ embedding. The second and third ways of adding subgraph $H$ both add two vertices and three edges, while decreasing the number of faces in the configuration by one, for a net change of -2 to the Euler characteristic and a genus increment of one. We see also that two face-boundary walks are incident on the new rung, which is type- $s$. We summarize this analysis by the production

$$
d_{j}\left(L_{n}\right) \rightarrow 2 d_{j}\left(L_{n+1}\right)+2 s_{j+1}\left(L_{n+1}\right) .
$$

Row (s) of Figure 2 yields the production

$$
s_{j}\left(L_{n}\right) \rightarrow 4 d_{j}\left(L_{n+1}\right) .
$$

From these two productions, it follows that the partial genus polynomials $D_{L_{n}}(z)$ and $S_{L_{n}}(z)$ satisfy the simultaneous recursion

$$
\begin{aligned}
D_{L_{0}}(z) & =1 \text { and } S_{L_{0}}(z)=0 \\
D_{n+1}(z) & =2 D_{n}(z)+4 S_{n}(z) \text { for } n \geq 2 \\
S_{n+1}(z) & =2 z D_{n}(z) \quad \text { for } n \geq 2, \text { with } \\
\Gamma_{L_{n}}(z) & =D_{n}(z)+S_{n}(z)=\sum_{j=0}^{\lfloor n / 2\rfloor} g_{i}\left(L_{n}\right) z^{n}
\end{aligned}
$$

Using the PGD-vector $V_{n}(z)=\left[D_{n}(z) S_{n}(z)\right]^{T}$ (where PGD stands for "partial-genus distribution") and a production matrix $M(z)$, we have

$$
M(z)=\left[\begin{array}{cc}
2 & 4 \\
2 z & 0
\end{array}\right] \quad V_{1}(z)=\left[\begin{array}{l}
1 \\
0
\end{array}\right]
$$

and this matrix form of the simultaneous recursion

$$
V_{n+1}(z)=M(z) V_{n}(z), \text { for } n \geq 1
$$

Applying the recursion yields these polynomials for the ladders $L_{1}, L_{2}, \ldots, L_{5}$.

\begin{tabular}{cccc}
$n$ & $D_{L_{n}}(z)$ & $S_{L_{n}}(z)$ & $\Gamma_{L_{n}}(z)$ \\
\hline 1 & 1 & 0 & 1 \\
2 & 2 & $2 z$ & $2+2 z$ \\
3 & $4+8 z$ & $4 z$ & $4+12 z$ \\
4 & $8+32 z$ & $8 z+16 z^{2}$ & $8+40 z+16 z^{2}$ \\
5 & $16+96 z+64 z^{2}$ & $16 z+64 z^{2}$ & $16+112 z+128 z^{2}$
\end{tabular}

Solution to this recursion, given by [19], is

$$
g_{j}\left(L_{n}\right)= \begin{cases}2^{n-1-j}\left(\begin{array}{c}
n+1-j \\
j
\end{array}\right) \frac{2 n+2-3 j}{n+1-j} & \text { for } j \leq\left\lfloor\frac{n+1}{2}\right\rfloor \\
0 & \text { otherwise }\end{cases}
$$

An application of the Cayley-Hamilton theorem in [12] leads to a method to derive a single recursion for the genus polynomials of the graphs in a linear sequence, rather than simultaneous recursions. In that single recursion the coefficients of $z^{n}$ are polynomials in $z$, whose degrees might possibly be as large as the number of different embedding types in a partition. A highly general formal definition of linear sequence of graphs is also given by [12].

\section{$3.2 \quad$ Ring-like graphs}

As depicted in Figure 1, a ring-like graph is obtained from a graph $G_{n}$ in an $H$-linear sequence by identifying some subgraph in the graph $G_{0}$ to an isomorphic subgraph in the iterated graph $H$, which is called a selfamalgamation on $G_{n}$. Figure 3 illustrates three different ways of constructing a ring-like sequence from ladders.

In Row (a) of Figure 1, the two end-rungs of the ladder $L_{3}$ are thickened to indicate that they will be amalgamated. If they are amalgamated without a twist, the result is the circular ladder $C L_{3}$. With a twist, the result is the Möbius ladder $M L_{3}$. Formulas for the genus polynomials of circular ladders and Móbius ladders were first derived by [47]. In Row (b), the graph $L_{0}^{\prime}$ replaces $L_{0}$ as the initial graph, and amalgamating the two 
(a)

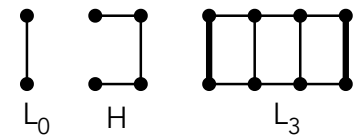

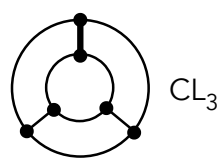

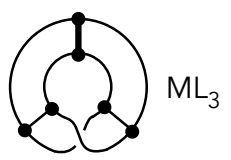

(b)
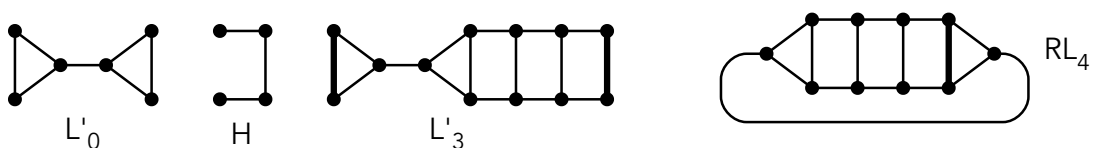

Figure 3: The circular ladder $C L_{3}$, the Möbius ladder $M L_{3}$, and the Ringel ladder $R L_{4}$ as ring-like constructions.

thickened subgraphs in $L_{3}^{\prime}$ yields the Ringel ladder $R L_{4}$. The graphs $R L_{n}$ were introduced by Gustin [38] as current graphs and used to spectacular advantage by Ringel and Youngs [55] in their solution to the Heawood map-coloring problem. A formula for the genus polynomials of Ringel ladders was derived by [60].

The effects of the topological operation of self-amalgamating a graph $G$ on a single vertex and on a single edge on the genus polynomial of $G$ are derived by [20] and [52], respectively. A more general study of the effects on genus polynomials and crosscap polynomials of the conversion from linear sequences to ring-like sequences is given by [7].

We recall (e.g., [56]) that Chebyshev polynomials of the second kind are defined, for $n \geq 0$, by the formula

$$
U_{n}(\cos \theta)=\frac{\sin (n+1) \theta}{\sin \theta} .
$$

Equivalently, $U_{n}(x)$ is a polynomial of degree $n$ in $z$ with integer coefficients, given by the recurrence

$$
\begin{aligned}
U_{0}(z) & =1 \\
U_{1}(z) & =2 z, \text { and } \\
U_{n}(z) & =2 z U_{n-1}(z)-U_{n-2}(z) .
\end{aligned}
$$

We further recall that the generating function for the Chebyshev polynomials is given by

$$
\sum_{n \geq 0} U_{n}(z) t^{n}=\frac{1}{1-2 z t+t^{2}}
$$

Using Chebyshev polynomials, the genus polynomials of Ringel ladders and of circular ladders were rederived by [34] and [10], respectively, and the log-concavity of the polynomials in both sequences was established.

The non-orientable embedding distributions for ladders and a couple of other sequences of graphs were derived by [5] by using the rank of the overlap matrix of [48]. Non-orientable embedding distributions for Ringel ladders were obtained by [15].

\section{Non-linear families}

There are two kinds of non-linear families of graphs whose genus polynomials are known. In a family of the first kind, the graphs typically have only one or two vertices of increasing valence. A member graph of four of these families is shown in Figure 4. Discussion of families of the second kind appears in $\S 4.2$.

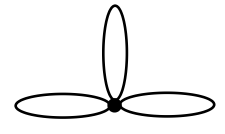

bouquet $\mathrm{B}_{3}$

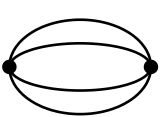

dipole $\mathrm{D}_{4}$

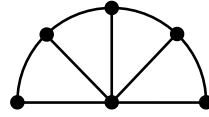

fan $\mathrm{F}_{5}$

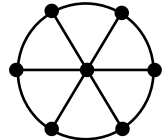

wheel $\mathrm{W}_{6}$

Figure 4: A bouquet, a dipole, a fan, and a wheel.

\subsection{One or two vertices of high valence}

The genus polynomials for several families of graphs with only one or two vertices of increasing valence have been calculated by counting the number of certain kinds of permutations with a given number of cycles. We denote the symmetric group on $m$ symbols by $\Sigma_{m}$. We denote the number of cycles of a permutation $\pi$ by $|\pi|$. 
The graph with one vertex and $n$ loops (like the leftmost graph in Figure 4) is called a bouquet and is denoted by $B_{n}$. We label the edges of $B_{n}$ by the integers $1,2, \ldots, n$, and for $1 \leq k \leq n$, we label the half edges of edge $k$ by $k^{+}$and $k^{-}$. Each cycle of the half-edges of $B_{n}$ represents a rotation system of $B_{n}$. Each cycle of the composition of a rotation system $\rho$ with the full involution

$$
\lambda=\left(1^{+} 1^{-}\right)\left(2^{+} 2^{-}\right) \cdots\left(n^{+} n^{-}\right)
$$

traces the boundary walk of a face of the embedding. Thus, the number of faces of the corresponding embedding is $|\rho \lambda|$.

Starting with the Euler polyhedral formula $|V|-|E|+|F|=2-2 \gamma$, we calculate that the number of faces in an embedding of $B_{n}$ of genus $j$ is $n+1-2 j$. We combine these observations by this theorem.

Theorem 4.1. The number $g_{j}\left(B_{n}\right)$ of embeddings of the bouquet $B_{n}$ of genus $j$ equals the number of permutations $\pi \in \Sigma_{2 n}$ such that $|\pi \lambda|=n+1-2 j$.

Based on a formula that Jackson [42] obtained by using group character theory, Theorem 4.2 was derived by [36], where Table 1 also appears.

Theorem 4.2. The numbers $g_{j}\left(B_{n}\right)$ satisfy the recurrence

$$
\begin{aligned}
(n+1) g_{j}\left(B_{n}\right)= & 4(2 n-1)(2 n-3)(n-1)^{2}(n-2) g_{j-1}\left(B_{n-2}\right) \\
& +4(2 n-1)(n-1) g_{j}\left(B_{n-1}\right), \text { for } n \geq 3
\end{aligned}
$$

with the initial conditions

$$
\begin{gathered}
g_{j}\left(B_{n}\right)=0 \text { if } j<0 \text { or } n<0, \\
g_{0}\left(B_{0}\right)=g_{0}\left(B_{1}\right)=1 \text { and } g_{j}\left(B_{0}\right)=g_{j}\left(B_{1}\right)=0 \text { for } j \geq 1, \\
g_{0}\left(B_{2}\right)=4, g_{1}\left(B_{2}\right)=2, \text { and } g_{j}\left(B_{2}\right)=0 \text { for } j \geq 2 .
\end{gathered}
$$

Table 1: Some values of $g_{j}\left(B_{n}\right)$.

\begin{tabular}{crrrr}
\hline$g_{j}\left(B_{n}\right)$ & $j=0$ & 1 & 2 & total \\
\hline$n=0$ & 1 & & & $1=0 !$ \\
1 & 1 & & & $1=1 !$ \\
2 & 4 & 2 & & $6=3 !$ \\
3 & 40 & 89 & & $120=5 !$ \\
4 & 672 & 3,360 & 1,008 & $5,040=7 !$ \\
5 & 16,128 & 161,280 & 185,452 & $362,880=9 !$ \\
\hline
\end{tabular}

The dipole $D_{n}$ is the graph with two vertices that are joined by $n$ edges, as shown second from the left in Figure 4. A recursion for its genus polynomials was derived using group characters both by [54] and [45]. A closed formula for a special case is given by [63]. Polynomials are given by [29] for a generalization of dipoles that allows a single loop at either or both vertices.

The fan graph $F_{n}$ is obtained by joining a single vertex $u$ to the path graph $P_{n}$ with $n$ vertices $v_{1}, v_{2}, \ldots, v_{n}$. The graph $F_{5}$ is third from the left in Figure 4. The fan graphs were generalized by [14] so as to allow replacement of the edge joining $u$ with the vertex $v_{j}$ by a multi-edge of multiplicity $t_{j}$, for $1 \leq j \leq n$, and their total embedding distributions were calculated.

The wheel graph $W_{n}$ (often, wheel), shown rightmost in Figure 4, is obtained by joining a single vertex with the vertices in the $n$-cycle graph $C_{n}$. A multi-wheel is obtained from a wheel by replacing any (or all) of its spokes by multi-edges. The region-number distributions of wheels was derived by [11], along with the region-number distributions for multi-wheels and for several other generalizations of wheels that have only one vertex of increasing valence, using a formula of [65].

Non-orientable distributions for bouquets and dipoles were derived by [46].

\subsection{Planar graphs of low treewidth}

In a non-linear family of known genus distribution of the second kind, the graphs are of low maximum valence and low treewidth. Three such families, all planar, are illustrated in Figure 5. Rather than a closed formula or a recursion for their genus distributions, we have quadratic-time algorithms.

An outerplanar graph is a graph $G$ that has an embedding in the sphere $S_{0}$, in which the boundary-walk of some face that we will call $f_{\infty}$ contains every vertex of $G$. Then $f_{\infty}$ is called the exterior face, and the fixed embedding represented is called an outerplane embedding. 


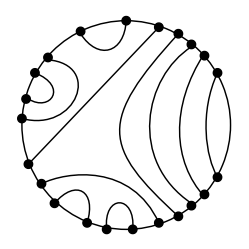

cubic outerplanar

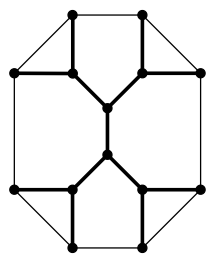

cubic Halin graph

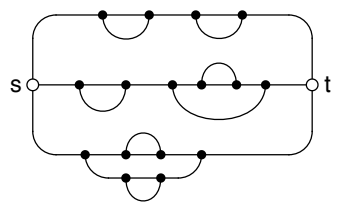

cubic series-parallel graph

Figure 5: A cubic outerplanar graph, a cubic Halin graph, and a cubic series-parallel graph.

The algorithm [21] for the genus polynomial of a cubic outerplanar graph $G$ is inspired by the observation regarding the Poincaré dual of an outerplane embedding, that when the region $f_{\infty}$ and the dual vertex within it are deleted, what remains of the outerplane embedding is a disk subdivided into simply connected regions, and what remains of the dual graph is a tree $T$. We may choose any vertex of $T$ as its root vertex. The algorithm proceeds by reassembling these regions according to a post-order traversal of the tree. The genus polynomial is calculated by iterative application of the productions derived by [50] for the effect of amalgamating two graphs $G_{1}$ and $G_{2}$ along an edge that has 2-valent endpoints in each of the amalgamands.

A Halin graph [40] is constructed from a plane tree $T$ with at least four vertices and no 2-valent vertices by drawing a cycle thru the leaves of $T$ in the order they occur in a preorder traversal of $T$. The outer cycle of a Halin graph is the cycle corresponding to the traversal of the leaves of the inscribed tree. In the Halin graph of Figure 5, the outer cycle has length eight.

The algorithm [22] for the genus polynomial of a cubic Halin graph begins by drawing the Halin graph embedding so that the edges are straight lines and so that the complement of the exterior face is a convex polygon. Roughly, here's how the algorithm continues. A new vertex is inserted at the midpoint of each edge of the boundary cycles of the polygon, and every region of the polygon is triangulated by drawing new edges from its midpoint vertex to other vertices on the boundary of the region. Each such triangle is paired with the unique triangle which it meets along a tree-edge, thereby forming a quadrangle. Then the polygon is reassembled by iteratively pasting three quadrangles together at a tree-vertex. There are productions derived for the effect on the genus polynomial of each such 3-way merger.

A series-parallel graph $G$ is specified via a recursively defined structure $(G, s, t)$, in which the graph $G$ has two distinct vertices $s$ ("source") and $t$ ("target").

- The graph $K_{2}$ with vertices $s$ and $t$ is a series-parallel graph.

- If $\left(G_{1}, s_{1}, t_{1}\right)$ and $\left(G_{2}, s_{2}, t_{2}\right)$ are disjoint series-parallel graphs, then so is the graph formed from them by (series operation) amalgamating vertices $t_{1}$ and $s_{2}$, and then using $s_{1}$ and $t_{2}$ as source and target or by (jackknife operation) amalgamating vertices $t_{1}$ and $s_{2}$, and then using $s_{1}$ and $t_{1}$ as source and target.

- If $\left(G_{1}, s_{1}, t_{1}\right)$ and $\left(G_{2}, s_{2}, t_{2}\right)$ are disjoint series-parallel graphs, then so is the graph formed from them by (parallel operation) amalgamating vertices $s_{1}$ and $s_{2}$ as new source and amalgamating vertices $t_{1}$ and $t_{2}$ as new target.

Once again, the genus polynomial is calculated by a reassembly algorithm [25].

\subsection{Automated calculation for bounded treewidth}

Since calculating the minimum genus of a graph is an NP-hard problem [61], even for cubic graphs [62], it follows that calculating genus polynomials is NP-hard. However, bounding the treewidth has been shown to lead to polynomial-time algorithms for minimum genus and for genus polynomials.

The usual definition of treewidth is based on the concept of tree decomposition. These concepts are both due to Robertson and Seymour [57]. An excellent exposition is given by [2]. For applications of treewidth to topological graph theory, see [49]. We now proceed with the definition of treewidth.

Let $G$ be a graph and $T$ a tree with nodes $1,2, \ldots, s$. Let $\Xi=\left\{X_{i} \mid 1 \leq i \leq s\right\}$ be a family of subsets of $V(G)$ (associated with the respective nodes $1,2, \ldots, s$ ) whose union is $V(G)$ such that

- the induced graph on the set of images in the tree $T$ of each vertex of $V(G)$ is a subtree of $T$;

- for every edge $u v$ in the graph $G$, there is a node $i$ in the tree $T$ such that both $u$ and $v$ are members of $X_{i}$.

Then the pair $(\Xi, T)$ is called a tree decomposition of $G$, and the tree $T$ is called a decomposition tree for $G$. The width of a tree decomposition $(\Xi, T)$ equals

$$
\max \left\{\left|X_{i}\right||1 \leq i \leq| V_{T} \mid\right\}-1
$$


The treewidth of a graph $G$ is the smallest $k$ such that $G$ has a tree decomposition of width $k$.

For graphs of fixed treewidth and bounded valence, a quadratic-time algorithm is given by [23]. A linear-time algorithm for the minimum genus of a graph of bounded treewidth is given by [44]. Describing the recursive step (of adding a copy of the iterated subgraph $H$ ) in the construction of the graphs in a linear sequence by the addition of a set of paths led to an algorithm [26] for automated calculation of the production matrix whose execution time is proportional to the square of the number of embedding types.

\subsection{Digraph embeddings}

An Eulerian digraph is a digraph that has a directed Eulerian circuit that traverses every edge, or equivalently, such that at every vertex, the in-valence and the out-valence are equal.

A cellular embedding of an Eulerian digraph $D$ into a closed surface is said to be a directed embedding if the boundary of each face is a directed closed walk in $D$. The directed genus polynomial of an Eulerian digraph $D$ is the polynomial

$$
\Gamma_{D}(x)=\sum_{h \geq 0} g_{h}(D) x^{h}
$$

where $g_{h}(D)$ is the number of directed embeddings into the orientable surface $S_{h}$ of genus $h$, for $h=0,1, \ldots$ - The sequence $\left\{g_{h}(D) \mid h \geq 0\right\}$, which is called the directed genus distribution of the digraph $D$, is known for very few classes of graphs, compared to the genus distribution of a graph.

Some fundamental results were established by [3]. They include an interpolation theorem, that if there are directed embeddings of the digraph $D$ in the surfaces $S_{p}$ and $S_{q}$ and if $p \leq r \leq q$, then there is a directed embedding of $D$ in $S_{r}$. They also show that the gap between the minimum directed genus and the maximum directed genus of a digraph can be arbitrarily large. Obstructions to planarity were derived by [4]. The directed genus of the deBruijn graph was obtained by [39].

\section{Log-concavity and other possible attributes}

A polynomial $a_{0}+a_{1} z+a_{2} z^{2}+\cdots+a_{n} z^{n}$ is said to be $\log$-concave if the inequality

$$
a_{j-1} a_{j+1} \leq a_{j}^{2}
$$

holds for $1 \leq j \leq n-1$. The following conjecture [36], which has been affirmed for many graphs, is known as the Log-Concavity Conjecture for Genus Distributions (or by the initials $L C G D$ ) is now over 30 years old:

Conjecture 5.1 (LCGD). Every graph has a log-concave genus distribution.

An easily derived variant definition of the log-concavity of a sequence is that the successive ratios of consecutive elements of the sequence are non-increasing. Proof that the genus polynomials for ladders are logconcave [19] was obtained by applying this variant to a closed form for the coefficients of the genus polynomial. Proof that the genus polynomials for bouquets are log-concave [36] follows inductively from the definition and the recurrence (8).

A well-known theorem of Newton asserts that if the coefficents of a polynomial are non-negative and all the roots are real, then the polynomial is log-concave. It is proved by [59] that the genus polynomials derived in [19] and [36] are real-rooted and then conjectured, after examination of a few more genus polynomials, that all genus polynomials are real-rooted. However, a counterexample was found by [13].

Whereas the product $p(z) q(z)$ of two log-concave polynomials $p(z)$ and $q(z)$ is log-concave, it is easy to construct examples in which the sum $p(z)+q(z)$ is not log-concave. To expand the range of methods of establishing the log-concavity of genus polynomials, relations called synchronicity and ratio-dominance were introduced by [33] as an aid to proving the log-concavity of linear combinations that correspond to some frequently used graph operations.

Two non-negative sequences $A$ and $B$ are said to be synchronized, denoted by $A \sim B$, if both are log-concave and if they satisfy the inequalities

$$
a_{j-1} b_{j+1} \leq a_{j} b_{j} \text { and } a_{j+1} b_{j-1} \leq a_{j} b_{j} \text { for all } j .
$$

The following theorem of [33] is fundamental.

Theorem 5.1. Let $A$ and $B$ be synchronized sequences, and let $u, v>0$. Then the sequence $u A+v B$ is log-concave. 
Let $A=\left(a_{j}\right)$ and $B=\left(b_{j}\right)$ both be non-negative synchronized sequences. Then $B$ is said to be ratiodominant over $A$, denoted by $B \gtrsim A$ or $A \lesssim B$, if

$$
a_{j+1} b_{j} \leq a_{j} b_{j+1} \text { for all } j .
$$

That the convolution operator $*$ preserves log-concavity is well-known. The following theorem is used in proofs of log-concavity of the genus polynomials of graphs that are constructed by amalgamating two different graphs.

Theorem 5.2 ( [33]). Let $A, B, C, D$ be four non-negative log-concave sequences without internal zeros, such that $A \lesssim B$ and $C \lesssim D$. Then the convolution sequences $A * D$ and $B * C$ are synchronized.

The directed genus distribution of a 4-regular outerplanar digraph was derived and proved by [8] to be log-concave. Indeed, the corresponding genus polynomial is real-rooted.

\section{Monodromy and Ribbon graphs}

The flags of an embedded graph $G$ are the faces of a barycentric subdivision of the embedding. We observe that every flag is 3 -sided and that there are four flags incident on every edge of $G$. The canonical flag-labeling convention has a couple of uncomplicated rules, introduced in [31], for labeling the edges and flags of an embedded graph $G$ with $n$ edges. These rules simplify the algorithms for computations.

1. Label the edges of $G$ by the integers $1,2, \ldots, n$.

2. Label the flags incident on edge $j$ by the integers $4 j-3,4 j-2,4 j-1,4 j$, so that flag $4 j-2$ is on the same side of edge $j$ as flag $4 j-3$ and so that flag $4 j$ lies immediately opposite flag $4 j-3$ on the other side of edge $j$.

Figure 6 illustrates this configuration. Artifacts of the barycentric subdivision are shown with broken straightedges and hollow vertices.

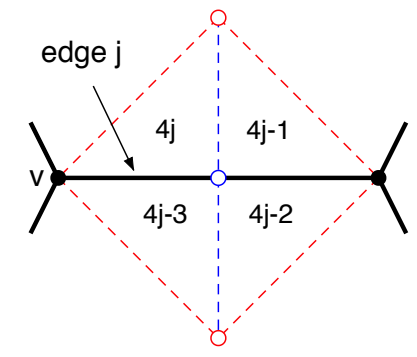

Figure 6: The canonical labeling of the four flags incident on edge $j$.

We now proceed with descriptions of the three paradigms mentioned in Section 1 for specifying a graph embedding: rotation systems, monodromy, and ribbon graphs.

\subsection{Monodromy}

Jones and Singerman [43] showed how a graph embedding could be specified by a set of three fixed-point-free involutions $\left\{r_{0}, r_{1}, r_{2}\right\}$, called the monodromy of the embedding, on the set of flags. The group that they generate is called the monodromy group. Using the canonical labeling of the flags, the involutions $r_{0}$ and $r_{2}$ for any embedded graph with $n$ edges are always the following:

$$
\begin{aligned}
& r_{0}=(12)(34)(56)(78) \cdots(4 n-34 n-2)(4 n-14 n) \\
& r_{2}=(14)(23)(58)(67) \cdots(4 n-34 n)(4 n-24 n-1) \text {. }
\end{aligned}
$$

To describe the involution $r_{1}$, we observe that the flags of any graph embedding are partitionable into cycles in which two consecutive flags lie in the same face of the embedding, with two flags in each corner of every face of the corresponding map. We define $r_{1}$ as the full involution that transposes every flag with the other flag in the same corner of the map. It should be clear that if we visualize each transposition in any of these involutions as a rule for pasting two flags together, we can reconstruct the embedding from its monodromy.

Proposition 6.1. Let $\left\{r_{0}, r_{1}, r_{2}\right\}$ be the monodromy of a graph embedding, and let $\lambda=r_{0} r_{2}$. Then the Poincaré dual embedding has the monodromy

$$
\left\{r_{0}^{*}=r_{0} \lambda, \quad r_{1}^{*}=r_{1}, \quad r_{2}^{*}=r_{2} \lambda\right\} .
$$

We observe that under the canonical labeling convention, we have

$$
\lambda=\left(\begin{array}{lll}
1 & 3
\end{array}\right)(24)(5 \quad 7)\left(\begin{array}{llll}
6 & 8
\end{array}\right) \cdots(4 n-34 n-1)(4 n-24 n) .
$$




\subsection{Ribbon graphs}

Formally, a ribbon graph, as defined by [53], is equivalent to a band decomposition as defined by [37], that is, a collection of disks and bands assembled as in Figure 7. Topologically, it is a surface with holes. The graph that it specifies is obtained by envisioning a vertex in the interior of each disk and an edge traversing each band, which joins the vertices in the disks at the opposite ends of the band. Any such ribbon graph has an implicit imbedding that is obtained by attaching the boundary of a disk to each of the boundary components. In discussion of ribbon graphs, including in this paper, the same phrase "ribbon graph" may refer to the implicit graph embedding.

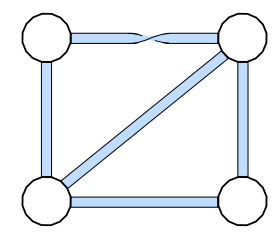

Figure 7: A ribbon graph whose embedding surface is the projective plane.

\section{Partial dualities}

In this section, we define and discuss Petrie duality and Wilson duality. We recall from Subsection 6.1 that the Poincaré duality operator is denoted by $*$. Propositions 6.1 and 7.1 give the transformation on the monodromy of a ribbon graph $G$ corresponding to $*$-duality and partial $*$-duality, respectively. The Petrie and Wilson duality operators are denoted by $\times$ and $* \times *$, respectively.

Chmutov [16] invented a way to define the Poincaré partial-dual of a ribbon graph $G$, in which one can dualize any edge-subset $A$ of $G$, in terms of a topological modification of the ribbon graph $G$. Since we denote the Poincaré dual of $G$ by $G^{*}$, we denote the partial-dual on $A$ by $\left.G^{*}\right|_{A}$. Although the full Poincaré dual has the same surface as the primal graph, partial-dualizing can change the surface type. For instance, partial-dualizing on one edge of a 2-cycle $C_{2}$ in the sphere $S_{0}$ results in an embedding of the bouquet $B_{2}$ (a single vertex with two loops) in the torus $S_{1}$. This partial-dual construction was extensively developed by [18].

To define $G^{\left.*\right|_{A}}$ in terms of permutation algebra, we begin with the definition

$$
\left.\lambda\right|_{A}=\prod_{j \in A}(4 j-34 j-1)(4 j-24 j)
$$

of $\left.\lambda\right|_{A}$ as the restriction of $\lambda$ to the edge-set $A$. This facilitates the computationally tractable definition of the partial dual of the embedded graph with monodromy $\left\{r_{0}, r_{1}, r_{2}\right\}$, as the embedded graph with monodromy

$$
\left\{r_{0}^{\left.*\right|_{A}}=\left.r_{0} \lambda\right|_{A}, r_{1}^{\left.*\right|_{A}}=r_{1}, r_{2}^{\left.*\right|_{A}}=\left.r_{2} \lambda\right|_{A}\right\}
$$

Proposition 7.1. The monodromy transformation given by (14) is equivalent to Chmutov's geometric construction of a partial-* dual.

The partial-dual genus polynomial ${ }^{\partial} \boldsymbol{\Gamma}_{\boldsymbol{G}}^{*}(\boldsymbol{z})$, for the Poincaré dual, as introduced by [30], is given by

$$
{ }^{\partial} \Gamma_{G}^{*}(z)=\sum_{A \subset E(G)} z^{\gamma\left(G^{* \mid A}\right)}
$$

The Petrie dual was originally conceived as a geometric construction. Its generalization to an operation on embedded graphs, and also the corresponding partial dual, are easily expressed in terms of a ribbon graph. The Petrie duality operator is indicated by $x$.

- The Petrie dual of a ribbon graph $G$ is the ribbon graph $G^{\times}$obtained by giving every ribbon a half-twist. Of course, the Petrie dual of an orientable ribbon graph may be non-orientable.

- The partial Petrie dual of a ribbon graph $G$ on an edge set $A$ is the ribbon graph $G^{\times\left.\right|_{A}}$ obtained by giving a half-twist to each ribbon whose corresponding edge lies in the edge-set $A$.

Proposition 7.2. Let $\left\{r_{0}, r_{1}, r_{2}\right\}$ be the monodromy of a graph embedding, and let $\lambda=r_{0} r_{2}$. Then the Petrie dual embedding has the monodromy

$$
\left\{r_{0}^{\times}=r_{0} r_{2}, \quad r_{1}^{\times}=r_{1}, \quad r_{2}^{\times}=r_{2}\right\} .
$$


Analogous to the definition (13) of $\left.\lambda\right|_{A}$, we now define

$$
\left.r_{2}\right|_{A}=\prod_{j \in A}(4 j-34 j)(4 j-24 j-1) .
$$

Proposition 7.3. Let $\left\{r_{0}, r_{1}, r_{2}\right\}$ be the monodromy of a graph embedding. Then the partial Petrie dual on the edge-subset $A$ has the monodromy

$$
\left\{r_{0}^{\times\left.\right|_{A}}=\left.r_{0} r_{2}\right|_{A}, \quad r_{1}^{\times\left.\right|_{A}}=r_{1}, \quad r_{2}^{\times\left.\right|_{A}}=r_{2}\right\}
$$

The Wilson dual is the iterated composition $* \times *$ of Poincaré and Petrie duals.

Proposition 7.4. Let $\left\{r_{0}, r_{1}, r_{2}\right\}$ be the monodromy of a graph embedding, and let $\lambda=r_{0} r_{2}$. Then the Wilson dual embedding has the monodromy

$$
\left\{r_{0}^{* \times *}=r_{0}, \quad r_{1}^{* \times *}=r_{1}, \quad r_{2}^{* \times *} s=r_{2} r_{0}\right\} .
$$

Analogous to the definitions (13) and (17), we now define

$$
\left.r_{0}\right|_{A}=\prod_{j \in A}(4 j-34 j-2)(4 j-14 j) .
$$

Proposition 7.5. Let $\left\{r_{0}, r_{1}, r_{2}\right\}$ be the monodromy of a graph embedding. Then the partial Wilson dual on the edge-subset $A$ has the monodromy

$$
\left\{r_{0}^{* \times\left. *\right|_{A}}=\left.r_{0} r_{2}\right|_{A}, \quad r_{1}^{* \times\left. *\right|_{A}}=r_{1}, \quad r_{2}^{* \times\left. *\right|_{A}}=r_{2}\right\}
$$

The following theorem indicates a close relationship between the partial- $* \times *$ and partial- $\times$ polynomials:

Theorem 7.1. Let $G$ be a ribbon graph. Then

$$
\partial \mathcal{E}_{G}^{* \times *}(z)=\partial_{\mathcal{E}}^{\times} G^{*}(z) .
$$

\section{Some partial-duality polynomial formulas}

Partial-dual polynomials formulas for a number of sequences of ribbon graphs were derived in [31]. Four of these sequences are represented by graphic images in Figure 8. These four sequences and two related sequences, both quite easily visualized, are listed immediately below the figure.

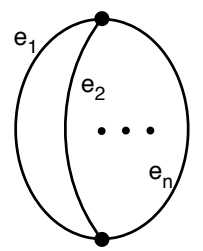

(2) $D_{n} \rightarrow S_{0}$

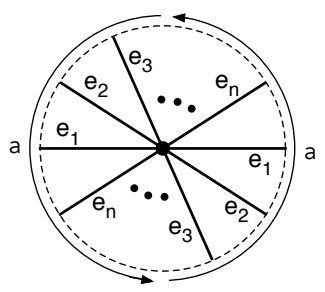

(5) $B_{n} \rightarrow N_{1}$

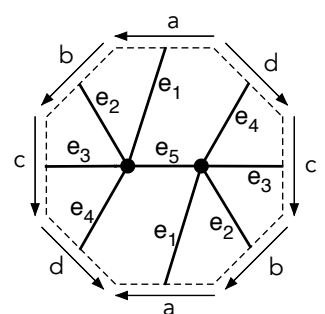

(3 odd) $\mathrm{D}_{5} \rightarrow \mathrm{S}_{2}$

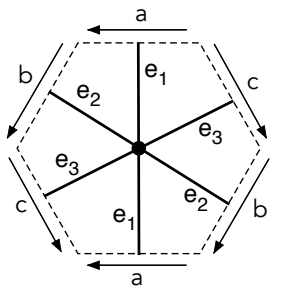

(6 odd) $\mathrm{B}_{3} \rightarrow \mathrm{S}_{1}$

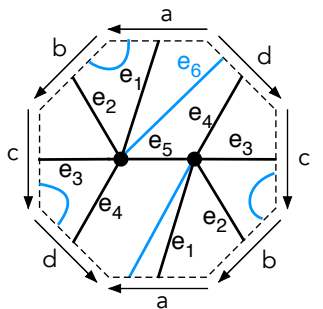

(3 even) $D_{6} \rightarrow S_{2}$

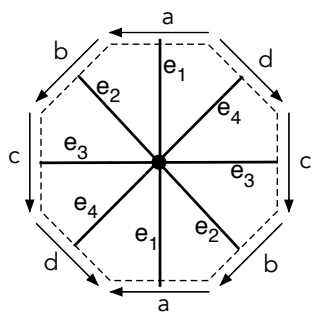

(6 even) $B_{4} \rightarrow S_{2}$

Figure 8: Representatives of some embedding sequences with known partial-dual polynomial formulas.

1. $C_{n} \rightarrow S_{0}$ is the $n$-cycle $C_{n}$ in the sphere $S_{0}$. 
2. $D_{n} \rightarrow S_{0}$ is the dipole $D_{n}$ in the sphere.

3. $D_{n} \rightarrow S_{p(n) / 2}$, where $p(n)= \begin{cases}n-1 & \text { if } n \text { odd } \\ n-2 & \text { if } n \text { even }\end{cases}$ is the dipole $D_{n}$ is the orientable surface of genus $p(n) / 2$.

4. $C_{n} \rightarrow N_{1}$ is the $n$ cycle in the projective plane $N_{1}$.

5. $B_{n} \rightarrow N_{1}$ is the $n$-bouquet in the projective plane.

6. $B_{n} \rightarrow S_{q(n) / 2}$, where $q(n)= \begin{cases}n-1 & \text { if } n \text { odd } \\ n & \text { if } n \text { even }\end{cases}$ is the bouquet $B_{n}$ in the orientable surface of genus $q(n) / 2$.

Table 2 gives the formulas for the partial-*, $\times$, and $* \times *$ polynomials for each of the first six sequences. For convenience, we refer to columns 3,4 , and 5 of that table according to the duality operator $*, \times, * \times *$ to which they correspond. We refer to a row according to its entry in column 1 . Thus, the notation $(2 \times)$ in row $(1)$, column $* \times *$ indicates (as we explain below) that the formula for the partial- $* \times *$ polynomials for the ribbon graph sequence $C_{n} \rightarrow S_{0}$ equals the formula in row (2), column $\times$, that is, the same as the formula for the partial- $\times$ polynomials for the ribbon graph sequence $D_{n} \rightarrow S_{0}$.

Table 2: Partial- $*, \times, * \times *$ polynomial formulas for six families of ribbon graphs. We define $p(n)=n-1$ for odd $n$ and $p(n)=n-2$ for even $n$. We define $q(n)=n-1$ for odd $n$ and $q(n)=n$ for even $n$.

\begin{tabular}{|c|c||c|c|c|}
\hline row & embedding & $\partial \mathcal{E}^{*}(z)$ & $\partial \mathcal{E}^{\times}(z)$ & $\partial^{* \times *}(z)$ \\
\hline \hline$(1)$ & $C_{n} \rightarrow S_{0}$ & $2+\left(2^{n}-2\right) z^{2}$ & $2^{n-1}(1+z)$ & $(2 \times)$ \\
\hline$(2)$ & $D_{n} \rightarrow S_{0}$ & " & $(1+z)^{n}-z^{n}+z^{p(n)}$ & $(1 \times)$ \\
\hline$\left(3_{\text {odd } n}\right)$ & $D_{n} \rightarrow S_{(n-1) / 2}$ & $2^{n} z^{n-1}$ & " & $(5 \times)$ \\
\hline$\left(3_{\text {even } n}\right)$ & $D_{n} \rightarrow S_{(n-2) / 2}$ & $2^{n-1}\left(z^{n-2}+z^{n}\right)$ & " & $(2 \times)$ \\
\hline$(4)$ & $C_{n} \rightarrow N_{1}$ & $2 z+\left(2^{n}-2\right) z^{2}$ & $(1 \times)$ & $(4 \times)=(1 \times)$ \\
\hline$(5)$ & $B_{n} \rightarrow N_{1}$ & " & $z(1+z)^{n}-z^{n+1}+z^{q(n)}$ & $(4 \times)=(1 \times)$ \\
\hline$\left(6_{\text {odd } n}\right)$ & $B_{n} \rightarrow S_{(n-1) / 2}$ & $\left(3_{\text {odd } n} *\right)$ & $"$ & $(2 \times)$ \\
\hline$\left(6_{\text {even } n}\right)$ & $B_{n} \rightarrow S_{n / 2}$ & $\left(3_{\text {even } n} *\right)$ & $"$ & $(5 \times)$ \\
\hline
\end{tabular}

As demonstrated in [31], these sequences are interrelated according to two principles:

(a) For each of the dualities $\bullet=*, \times, * \times *$, a ribbon graph and any of its partial- duals have the same partial-• polynomial.

(b) For any ribbon graph $G$, we have ${ }^{\partial \mathcal{E}_{G}^{*} \times *}(z)={ }^{\partial} \mathcal{E}_{G^{*}}^{\times}(z)$. This is Theorem 7.1.

For instance, the ribbon graph (2) $D_{n} \rightarrow S_{0}$ is the full $*$-dual of (1) $C_{n} \rightarrow S_{0}$, so by principle (a), they have the same partial-* polynomial. Moreover, since (2) $D_{n} \rightarrow S_{0}$ is the full $*$-dual of (1) $C_{n} \rightarrow S_{0}$, it follows from principle (b) that the partial-*×* polynomial of (1) $C_{n} \rightarrow S_{0}$ equals the partial-× polynomial of $(2) D_{n} \rightarrow S_{0}$.

\subsection{Ladders}

In this subsection, we present partial-*, $\times$, and $* \times *$ polynomials for the ladder graphs.

Theorem 8.1. [30] Let $p_{n}(z)={ }^{\partial} \Gamma_{L_{n}}^{*}(z)$ and $q_{n}(z)={ }^{\partial} \Gamma_{Q_{n}}^{*}(z)$. Then the resulting polynomials satisfy the simultaneous recurrence:

$$
\begin{aligned}
p_{n+1}(z) & =6 z p_{n}(z)+q_{n}(z) \\
q_{n+1}(z) & =\left(6 z+4 z^{2}\right) p_{n}(z)+(1+2 z) q_{n}(z)
\end{aligned}
$$

Theorem 8.2. [30] The polynomial ${ }^{\partial} \Gamma_{L_{n}}^{*}(z)$ for the ladders is given by

$$
{ }^{\partial} \Gamma_{L_{n}}^{*}(z)=2(\sqrt{8} z)^{n-1}\left(\frac{1+6 z+z^{2}}{2 \sqrt{2} z} U_{n-2}(t)-U_{n-3}(t)\right),
$$

where $t=\frac{1+8 z}{4 \sqrt{2} z}$ and $U_{m}(t)$ is the $m^{\text {th }}$ Chebyshev polynomial of the second kind. 
Theorem 8.3. [31] The ladder graph $L_{n}$ (embedded in the 2-sphere $S_{0}$, as shown in Figure 9 ) has the partial-× polynomial recursion

$$
\begin{aligned}
& \partial \mathcal{E}_{L_{0}}^{\times}(z)=2 \\
& \partial \mathcal{E}_{L_{1}}^{\times}(z)=8+8 z \\
& \partial \mathcal{E}_{L_{n}}^{\times}(z)=(2+4 z)^{\partial} \mathcal{E}_{L_{n-1}}^{\times}(z)+\left(16 z^{2}\right)^{\partial} \mathcal{E}_{L_{n-2}}^{\times}(z) \text { for } n \geq 2 .
\end{aligned}
$$

with the closed form

$$
{ }^{\partial} \mathcal{E}_{L_{n}}^{\times}(z)=2(4 i z)^{n}\left(\frac{1+z}{i z} U_{n-1}\left(\frac{1+2 z}{4 i z}\right)-U_{n-2}\left(\frac{1+2 z}{4 i z}\right)\right),
$$

where $i^{2}=-1$ and $U_{n}(t)$ is the $n^{\text {th }}$ Chebyshev polynomial. The polynomial ${ }^{\partial} \mathcal{E}_{L_{n}}^{\times}(z)$ is log-concave.

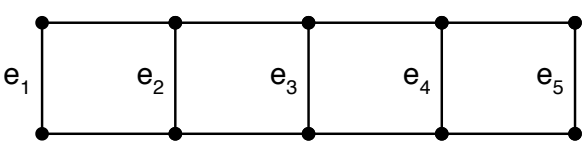

Figure 9: Embedding of the ladder $L_{5}$ in the sphere $S_{0}$.

Lemma 8.1. [31] Let $e$ be any edge of a ribbon graph $G$. Let $H$ be obtained from $G$ by adding an edge parallel to $e$ and then trisecting it by adding two vertices of valence two. Then

$$
\partial_{H}^{* \times *}(z)=\left(1+3 z+4 z^{2}\right)^{\partial} \mathcal{E}_{G}^{* \times *}(z) .
$$

Theorem 8.4. [31] The ladder $L_{n}$ has the partial- $* \times *$ polynomial

$$
{ }^{\partial} \mathcal{E}_{L_{n}}^{* \times *}=(1+z)\left(1+3 z+4 z^{2}\right)^{n} .
$$

Proof. The ladder $L_{0}$ consists of a single edge, so ${ }^{\partial} \mathcal{E}_{L_{0}}^{* \times *}=1+z$. Since $L_{n}$ is obtained from $L_{n-1}$ by adding a trisected parallel edge, this follows from Lemma 8.1.

\subsection{A family of series-parallel graphs}

The family of restricted series-parallel ribbon graphs, abbreviated $R S P G$, is recursively constructed, beginning with the trivial ribbon graph $K_{1}$. It is closed under the following operations: (1) adding a parallel edge or a trisected parallel edge; (2) ribbon-joins; (3) bar-amalgamations. All trees and ladders are in $R S P G$, but so are ribbon graphs like the one shown in Figure 10.

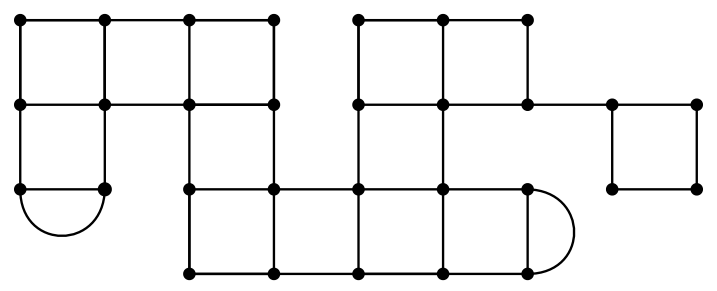

Figure 10: An RSPG graph that is not a tree or a ladder.

Theorem 8.5. [31] Let $G$ be any ribbon graph in $R S P G$. Then

$$
{ }^{\partial} \mathcal{E}_{G}^{* \times *}(z)={ }^{\partial \mathcal{E}_{G^{*}}} \times(z)=2^{k}(1+z)^{m}\left(1+3 z+4 z^{2}\right)^{n},
$$

where $k$ is the number of parallel edges, $m$ is the number of bar-amalgamations, and $n$ is the number of trisected parallel edges used in the construction of $G$.

\section{Some research problems}

We are concerned here with the derivation of methods for calculating genus polynomials and partial-duality polynomials for a number of graphs and embeddings of general interest. An attractive common feature of the examples we present in this section is that each of them has a plane embedding or a plane projection with circular symmetry. 


\subsection{Anti-prisms}

The $n$-anti-prism is the graph formed from two concentric $n$-gons so that each vertex of one of the $n$-gons are joined to the endpoints of an edge the other $n$-gon, as illustrated in Figure 11 . Each anti-prism graph is the 1-skeleton of the Archimedean solid from which its name is taken, in which all of the 3-sided faces are equilateral triangles.
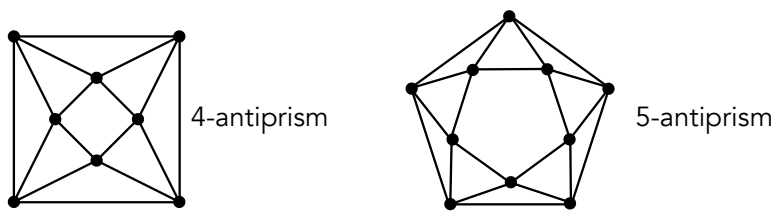

Figure 11: Two anti-prism graphs.

Problem 9.1. Derive the genus polynomials for the sequence of anti-prism graphs.

Problem 9.2. Derive the partial-dual polynomial of the spherically embedded anti-prism graphs for any or all of the dualities $*, \times, * \times *$.

\subsection{Truncated pyramid}

The truncated n-pyramid is the 1-skeleton of the solid formed by truncating all the corners of the pyramid with an $n$-gon as its base. (In some contexts other than graph theory, the apex of the pyramid would be truncated, but not the corners of its base.)
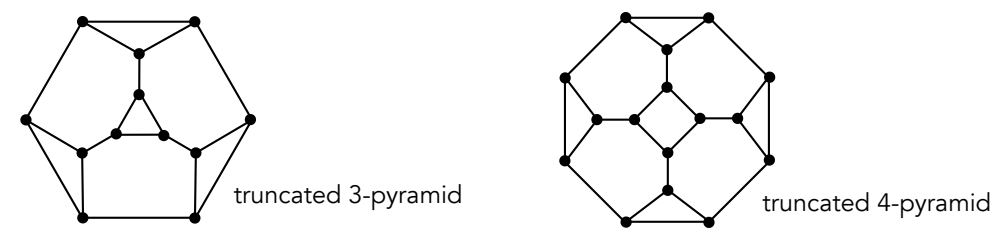

Figure 12: Two truncated-pyramid graphs.

Problem 9.3. Derive the genus polynomials for the sequence of truncated-pyramid graphs.

Problem 9.4. Derive the partial-dual polynomials of the spherically embedded truncated-pyramid graphs for any or all of the dualities $*, \times, * \times *$.

\subsection{Ring-like ladder graphs}

Problem 9.5. Derive the partial-dual polynomials of the spherically embedded ladder graphs for any or all of the dualities $*, \times, * \times *$.

Problem 9.6. Derive the partial-dual polynomials of the Möbius ladder graphs embedded in the projective plane $N_{1}$ for any or all of the dualities $*, \times, * \times *$.

\subsection{Circulant graphs}

A circulant $\operatorname{graph} \operatorname{circ}(n ; X)$ is defined for a positive integer $n$ and a subset $X$ of the integers $1,2, \ldots\lfloor n / 2\rfloor$, called the connections.

- The vertex set is $\mathbb{Z}_{n}$, the integers modulo $n$.

- There is an edge joining the vertices $i$ and $j$ if and only if the number $\mid j-i$ is one of the connections.

For instance, the graph $\operatorname{circ}(5,\{1,2\})$ is isomorphic to the Kuratowski graph $K_{5}$ (and not to the cicular ladder $\left.C L_{5}\right)$. Corresponding to the sequence of circulant graphs $\operatorname{circ}(n,\{1,2\})$ for $n \geq 5$, there is a sequence of toroidal embeddings, as shown in Figure 13.

Problem 9.7. Derive the partial- $*, \times, * \times *$ polynomials for the toroidally embedded circulant graphs $\operatorname{circ}(n,\{1,2\})$ for $n \geq 5$. 

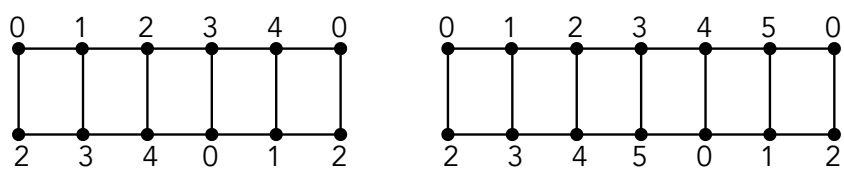

Figure 13: A sequence of toroidal embeddings of circulant graphs $\operatorname{circ}(5,\{1,2\}), \operatorname{circ}(6,\{1,2\}), \ldots$

\section{References}

[1] N.L. Biggs, E.K. Lloyd and R.J. Wilson, Graph Theory 1736-1936, Oxford University Press, 1976.

[2] H.L. Bodlaender, A partial k-arboretum of graphs with bounded treewidth, Theoret. Comput. Sci. 209 (1998), $1-45$.

[3] C.P. Bonnington, M. Conder, M. Morton and P. McKenna, Embedding digraphs on orientable surfaces, J. Combin. Theory Ser. B 85 (2002), 1-20.

[4] C.P. Bonnington, N. Hartsfield and J. Širáň, Obstructions to directed embeddings of Eulerian digraphs in the plane, European J. Combin. 25 (2004), 877-891.

[5] J. Chen, J.L. Gross and R.G. Rieper, Overlap matrices and total embedding distributions, Discrete Math. 128 (1994), 73-94.

[6] Y. Chen and J.L. Gross, An Euler-genus approach to the calculation of the crosscap-number polynomial, J. Graph Theory 88 (2018), 88-100.

[7] Y. Chen and J.L. Gross, Genus polynomials and crosscap-number polynomials for ring-like graphs, Math. Nachr. 292(4) (2019), 760-776.

[8] Y. Chen, J.L. Gross, and X. Hu, Enumeration of digraph embeddings, European J. Combin. 36 (2014), 660-678.

[9] Y. Chen, J.L. Gross and T. Mansour, Total embedding distributions of circular laddeers, J. Graph Theory $73(2)(2013), 32-57$.

[10] Y. Chen, J.L. Gross and T. Mansour, Log-concavity of genus distributions for circular ladders, Math. Nachr. 288(17-18) (2015), 1952-1969.

[11] Y. Chen, J.L. Gross and T. Mansour, On the genus distribution of wheels and of related graphs, Discrete Math. 341 (2018), 934-945.

[12] Y. Chen, J.L. Gross, T. Mansour and T.W. Tucker, Recurrences for the genus polynomials of linear sequences of graphs, Math. Slovaca 70 (2020), 505-526.

[13] Y. Chen and Y. Liu, On a conjecture of S. Stahl, Canad. J. Math. 62(5) (2010) 1058-1059.

[14] Y. Chen, T. Mansour and Q. Zou, Embedding distributions of generalized fan graphs, Canad. Math. Bull. 56 (2013), 265-271.

[15] Y. Chen, L. Ou and Q. Zou, Total embedding distributions of Ringel ladders, Discrete Math. 311 (2011), 2463-2474.

[16] S. Chmutov, Generalized duality for graphs on surfaces and the signed Bollobás-Riordan polynomial, J. Combin. Theory Ser. B 99 (2009), 617-638.

[17] J. Edmonds, A combinatorial representation for polyhedral surfaces (abstract), Notices Amer. Math. Soc. 7 (1960), 646.

[18] J. Ellis-Monaghan and I. Moffatt, Graphs on Surfaces: Dualities, Polynomials, and Knots, Springer, 2013.

[19] M. Furst, J.L. Gross and R. Statman, Genus distributions for two classes of graphs, J. Combin. Theory Ser. B 46 (1989), 523-534.

[20] J.L. Gross, Genus distribution of graph amalgamations: Self-pasting at root-vertices, Australas. J. Combin. 49 (2011), 19-38.

[21] J.L. Gross, Genus distribution of cubic outerplanar graphs, J. Graph Algorithms Appl. 15(2) (2011), 295316.

[22] J.L. Gross, Embeddings of cubic Halin graphs: genus distributions, Ars Math. Contemp. 6 (2013), 37-56.

[23] J.L. Gross, Embeddings of graphs of fixed treewidth and bounded degree, Ars Math. Contemp. 7 (2014), 379-403.

[24] J.L. Gross and M. Furst, Hierarchy for imbedding-distribution invariants of a graph, J. Graph Theory 11 (1987), 205-220.

[25] J.L. Gross, M. Kotrbcik and T. Sun, Genus distributions of cubic series-parallel graphs, Discrete Math. Theor. Comput. Sci. 16:3 (2014), 129-146. 
[26] J.L. Gross, I.F. Khan, T. Mansour and T.W. Tucker, Calculating genus polynomials via string operations and matrices, Ars Math. Contemp. 15 (2018), 267-295.

[27] J.L. Gross, I.F. Khan and M.I. Poshni, Genus distributions for iterated claws, Electron. J. Combin. 21(1) (2014), \#P1.12.

[28] J.L. Gross, I.F. Khan and M.I. Poshni, Genus distribution of graph amalgamations: Pasting at root-vertices, Ars Combin. 94 (2010), 33-53

[29] J.L. Gross, T. Mansour and T.W. Tucker, Valence-partitioned genus polynomials and their application to generalized dipoles, Australas. J. Combin. 67(2) (2017), 203-221.

[30] J.L. Gross, T. Mansour and T.W. Tucker, Partial duality for ribbon graphs, I: Distributions, European J. Combin. 86 (2020), 103084.

[31] J.L. Gross, T. Mansour and T.W. Tucker, Partial duality for ribbon graphs, II: partial-twuality polynomials and monodromy computations, manuscript, 2020.

[32] J.L. Gross, T. Mansour and T.W. Tucker, Partial duality for ribbon graphs, III: a Gray code algorithm for enumeration, manuscript, 2020.

[33] J.L. Gross, T. Mansour, T.W. Tucker and D.G.L. Wang, Log-concavity of combinations of sequences and applications to genus distributions, SIAM J. Discrete Math. 29(2) (2015), 1002-1029.

[34] J.L. Gross, T. Mansour, T.W. Tucker and D.G.L. Wang, Log-concavity of the genus polynomials of Ringel ladders, Electron. J. Graph Theory Appl. 3 (2015), 109-126.

[35] J.L. Gross, T. Mansour, T.W. Tucker and D.G.L. Wang, Iterated claws have real-rooted genus polynomials, Ars Math. Contemp. 10 (2016), 255-268.

[36] J.L. Gross, D.P. Robbins and T.W. Tucker, Genus distributions for bouquets of circles, J. Combin. Theory Ser. B 47 (1989), 292-306.

[37] J.L. Gross and T.W. Tucker, Topological Graph Theory. Wiley, 1987 (reprinted by Dover, 2001).

[38] W. Gustin, Orientable embedding of Cayley graphs, Bull. Amer. Math. Soc. 69 (1963), 272-275.

[39] A.W. Hales and N. Hartsfield, The directed genus of the de Bruijn graph, Discrete Math. 309 (2009), $5259-5263$.

[40] R. Halin, Über simpliziale Zerfällungen beliebiger (endlicher oder unendlicher) Graphen, Math. Ann. 156 (1964), 216-225.

[41] L. Heffter, Über das Problem der Nachbargebiete, Math. Ann. 38 (1891), 477-508.

[42] D.M. Jackson, Counting cycles in permutations by group characters, with an application to a topological problem, Trans. Amer. Math. Soc. 299 (1987), 785-801.

[43] G.A. Jones and D. Singerman, Theory of maps on orientable surfaces, Proc. Lond. Math. Soc. III 37 (1978), $273-307$.

[44] K. Kawarabayashi, B. Mohar and B. Reed, A simpler linear time algorithm for embedding graphs into an arbitrary surface and the genus of graphs of bounded tree-width, Proc. 49th Ann. Symp. on Foundations of Comput. Sci. (FOCS'08) IEEE (2008), 771-780.

[45] J.H. Kwak and J. Lee, Genus polynomials of dipoles, Kyungpook Math. J. 33 (1993), 115-125.

[46] J.H. Kwak and S.H. Shim, Total embedding distributions for bouquets of circles, Discrete Math. 248 (2002), 93-108.

[47] L.A. McGeoch, Algorithms for two graph problems: computing a maximum genus imbedding and the twoserver problem, $\mathrm{PhD}$ thesis, Carnegie-Mellon Univ., 1987.

[48] B. Mohar, An obstruction to embedding graphs in surfaces, Discrete Math. 78 (1989), 135-142.

[49] B. Mohar and C. Thomassen, Graphs on Surfaces, Johns Hopkins University Press, 2001.

[50] M.I. Poshni, I.F. Khan and J.L. Gross, Genus distribution of edge-amalgamations, Ars Math. Contemp. 3 (2010), 69-86.

[51] M.I. Poshni, I.F. Khan and J.L. Gross, Genus distribution of 4-regular outerplanar graphs, Electron. J. Combin. 18 (2011), \#P212.

[52] M.I. Poshni, I.F. Khan and J.L. Gross, Genus distribution of graphs under self-edge-amalgamations, Ars Math. Contemp. 5 (2012), 127-248.

[53] N. Reshetikhin and V. Turaev, Ribbon graphs and their invariants derived from quantum groups, Comm. Math. Phys. 127 (1990), 1-26.

[54] R.G. Rieper, The enumeration of graph imbeddings, Ph.D. Dissertation, Western Michigan University, 1990.

[55] G. Ringel and J.W.T. Youngs, Solution of the Heawood map-coloring problem, Proc. Nat. Acad. Sci. USA 60 (1968), 438-445.

[56] T. Rivlin, Chebyshev Polynomials. From Approximation Theory to Algebra and Number Theory, John Wiley, New York, 1990. 
[57] N. Robertson and P.D. Seymour, Graph minors. II, Algorithmic aspects of tree-width, J. Algorithms 7 (1986), 309-322.

[58] S. Stahl, Permutation-partition pairs. III. Embedding distributions of linear families of graphs, J. Combin. Theory B 52 (1991), 191-218.

[59] S. Stahl, On the zeros of some genus polynomials, Canad. J. Math. 49 (1997), 617-640.

[60] E.H. Tesar, Genus distributions of Ringel ladders, Discrete Math. 216 (2000), 235-252.

[61] C. Thomassen, The graph genus problem is NP-complete, J. Algorithms 10 (1989), 568-576.

[62] C. Thomassen, The genus problem for cubic graphs, J. Combin. Theory Ser. B 69 (1997), 52-58.

[63] T.I. Visentin and S.W. Wieler, On the genus distribution of ( $p, q, n)$-dipoles, Electron. J. Combin. 14 (2007), \#R12.

[64] A.T. White, Graphs of Groups on Surfaces, North-Holland, 2001.

[65] D. Zagier, On the distribution of the number of cycles of elements in symmetric groups, Nieuw Arch. Wiskd. 13(3) (1995), 489-495. 